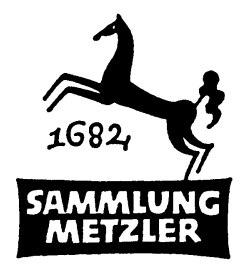

REALIEN ZUR LITERATUR

ABT. D:

LITERATURGESCHICHTE 
JURGEN SCHUTTE

\section{Lyrik des deutschen Naturalismus}

(1885-1893)

MCMLXXVI

J. B. METZLERSCHE VERLAGSBUCHHANDLUNG

STUTTGART 
ISBN 978-3-476-10144-0

ISBN 978-3-476-03854-8 (eBook)

DOI 10.1007/978-3-476-03854-8

\section{144}

(C) Springer-Verlag GmbH Deutschland 1976

Ursprünglich erschienen bei J. B. Metzlersche Verlagsbuchhandlung und Carl Ernst Poeschel Verlag GmbH in Stuttgart 1976 


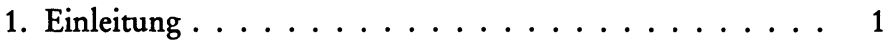

1.1. Naturalismus . . . . . . . . . . . . 3

1.2. Gesellschaftliche Entwicklung und literarische Intelligenz . . . . . . . . . . . . . 9

2. Umriß und Entwicklung der naturalistischen Lyrik . . . 13

2.1. Autoren, Abgrenzungen und Stellenwert . . . . . . 13

2.2. Der literarische und gesellschaftliche Anspruch der "jüngstdeutschen « Lyriker . . . . . . . . . . 22

2.3. Zur Charakteristik der "Modernen-Dichter-Charaktere« . . . . . . . . . . 29

2.4. Von der »Lyriker-Revolution« zur "Revolution der Lyrik« . . . . . . . . . . . . . . 40

3. Die Autoren . . . . . . . . . . . . . . . . 47

4. Zur Forschungslage . . . . . . . . . . . 73

5. Literaturverzeichnis . . . . . . . . . . . . 81

5.1. Anthologien und Dokumente . . . . . . . . . 81

5.2. Gesamtdarstellungen und Einzelaspekte . . . . . . . 8 81

5.3. Einzelne Autoren . . . . . . . . . . . . . . . . 88

6. Zeittafel zur naturalistischen Lyrik . . . . . . . . . 91

7. Namen- und Titelregister . . . . . . . . . . . 92

\section{VORBEMERKUNG}

Die vorliegende Arbeit ist im Zusammenhang mit dem an der Freien Universität Berlin seit 1973 laufenden Forschungsprojekt $*$ Literaturgeschichte und Sozialgeschichte 1880-1918 entstanden. Den Teilnehmern dieses Projekts, besonders Klaus R. Scherpe, danke ich für vielfältige Anregung und fördernde Kritik. Klaus Dieter Franke (Münster) gewährte mir freundlicherweise Einblick in seine umfangreiche, bisher nicht veröffentlichte Arbeit über Karl Henckell.

West-Berlin, Oktober 1975

J.S. 


\section{ABKURZUNGEN}

ADB Allgemeine Deutsche Biographie

AM Karl Henckell, Amselrufe. Gedichte.

Bode Ingrid Bode. Die Autobiographien zur deutschen Literatur, Kunst und Musik 1900 bis 1965. Bibliographie und Nachweise der persönlichen Begegnungen und Charakteristiken. Stuttgart 1966.

Brümmer Franz Brümmer, Lexikon der deutschen Dichter und Prosaisten von Beginn des 19. Jahrhunderts bis zur Gegenwart, 6. Aufl., 8 Bde, Leipzig 1913.

$\mathrm{BZ}$ Arno Holz, Buch der Zeit. Lieder eines Modernen.

DIO Karl Henckell, Diorama. Gedichte.

DLE Deutsche Literatur. Sammlung literarischer Kunst- und Kulturdenkmäler in Entwicklungsreihen. Hrsg. Heinz Kindermann, Walther Brecht und Dietrich Kralik. Leipzig $1928 \mathrm{ff}$.

DVjs Deutsche Vierteljahrsschrift für Literaturwissenschaft und Geistesgeschichte

Friedrichs Elisabeth Friedrichs, Literarische Lokalgrößen 1700-1900. Verzeichnis der in regionalen Lexika und Sammelwerken ausgeführten Schriftsteller. Stuttgart 1967.

Ges Die Gesellschaft, Jg. 1-18, 1885-1902. Reprint: Nendeln 1970.

GR Germanic Review

Int. Bibl. Internationale Bibliographie zur Geschichte der deutschen Literatur. Gesamtredaktion G. Albrecht und G. Dahle. Bd I; II, 1; II, 2. Berlin 1969-1972.

JEGP Journal for English and Germanic Philology

Kosch (2) Wilhelm Kosch, Deutsches Literatur-Lexikon. 2. Aufl., 4 Bde. Bern 1949-1958.

Kosch (3) Wilhelm Kosch, Deutsches Literatur-Lexikon, begr. von Wilhelm Kosch. 3. Aufl., Bd. 1-4 (Aal-Filchner). Bern $1961 \mathrm{ff}$.

L Literatur (Hinweis auf Titel im Literaturverzeichnis).

LSDL Lexikon sozialistischer deutscher Literatur. s'Gravenhage 1973 [Reprint].

Magazin Magazin für die Literatur des In- und Auslandes

MD Wilhelm Arent (Hrsg), Moderne Dichter-Charaktere

NDB Neue Deutsche Biographie

PMLA Publications of Modern Language Association

W/G Gero von Wilpert und Adolf Gühring, Erstausgaben deutscher Dichtung 1600-1960. Stuttgart 1967. 
So wie die Kriegsgeschichte nur die Namen von Generälen kennt, die vordem uneinnehmbare Festungen stürmten und auf ihnen ihre Fahnen aufpflanzten, feindliche Heere zurückdrängten und besiegten - so bucht auch die Literaturgeschichte auf die Dauer keine anderen Namen.

(Georg Hermann, Der tote Naturalismus, in: Literarisches Echo 15, 1912/13, Sp. 26).

Die jeweils Herrschenden sind aber die Erben aller, die je gesiegt haben. Die Einführung in den Sieger kommt demnach den Herrschenden allemal zugut. Damit ist dem historischen Materialisten genug gesagt. Wer immer bis zu diesem Tag den Sieg davontrug, der marschiert mit in dem Triumphzug, der die heute Herrschenden über die dahinführt, die heute am Boden liegen. Die Beute wird, wie das immer so üblich war, im Triumphzug mitgeführt. Man bezeichnet sie als die Kulturgüter. Sie werden im historischen Materialisten mit einem distanzierten Betrachter zu rechnen haben. Denn was er an Kulturgütern überblickt, das ist ihm samt und sonders von einer Abkunft, die er nicht ohne Grauen bedenken kann. Es dankt sein Dasein nicht nur der Mühe der großen Genien, sondern auch der namenlosen Fron ihrer Zeitgenossen. Es ist niemals ein Dokument der Kultur, ohne zugleich ein solches der Barbarei zu sein. Und wie es selbst nicht, in der es von dem einen an den andern gefallen ist. Der historische Materialist rückt daher nach Maßgabe des Möglichen von ihr ab. Er betrachtet es als seine Aufgabe, die Geschichte gegen den Strich zu bürsten.

(Walter Benjamin, Geschichtsphilosophische Thesen VII, in: Illuminationen, Frankfurt/M 1961, S. 271 f.). 